\title{
Impacto del control de la etiología de la cirrosis hepática en la evolución clínica
}

\section{Impact of control of the etiology of liver cirrhosis on clinical evolution}

\author{
Zucel D. Cruz-Hernández ${ }^{1 *}$, Marcia Samada-Suárez², Harlim Rodríguez-Rodríguez ${ }^{3}$, \\ Teresita Pérez-González ${ }^{3}$, Julio C. Hernández-Perera² y Lissette Chao-González² \\ ${ }^{1}$ Departamento de Gastroenterología, Hospital General Leopoldito Martínez, San José de Las Lajas, Mayabeque; ${ }^{2}$ Departamento de Gastroenterología \\ y Hepatología, Hospital Cimeq, Playa, La Habana; ${ }^{3}$ Departamento de Gastroenterología, Hospital General Iván Portuondo, San Antonio de Los \\ Baños, Artemisa. Cuba
}

\section{Resumen}

Antecedentes: La cirrosis hepática constituye uno de los principales problemas de salud en el mundo. La fase compensada de la enfermedad se relaciona con una larga supervivencia y la aparición de complicaciones determina la fase descompensada. El control de la etiología puede influir en la progresión de la enfermedad. Objetivo: Evaluar el impacto del control etiológico de la cirrosis en la evolución clínica de pacientes cirróticos. Método: Se realizó un estudio descriptivo, retrospectivo y longitudinal en pacientes con cirrosis compensada. Estos pacientes fueron evaluados en el Hospital Cimeq. Se estudiaron 48 pacientes. Las variables estudiadas fueron: progresión de las várices esofágicas y del tamaño del bazo, control de la etiología, fase de la enfermedad y supervivencia libre de complicaciones. Resultados: Presentaron control de la etiología 22 pacientes. De estos, 19 permanecieron en la fase compensada. El tiempo promedio de supervivencia libre de complicaciones mayores en los pacientes con etiología controlada fue de 17 años. Conclusiones: La progresión de la hipertensión portal y la permanencia de los pacientes en la fase compensada de la enfermedad se relacionaron con el control de la etiología. La mayor supervivencia libre de complicaciones fue significativamente mayor en los pacientes con control de la etiología de la cirrosis.

Palabras claves: Cirrosis hepática. Hipertensión portal. Control de la etiología.

\section{Abstract}

Background: Liver cirrhosis constitutes one of the main health problems in the world. The compensated phase of the disease is related to long survival, and the appearance of complications determines the decompensated phase. The control of the etiology can influence the progression of the disease. Objective: To evaluate the impact of the etiological control of cirrhosis on the clinical evolution of cirrhotic patients. Method: A descriptive, retrospective and longitudinal study was performed in patients with compensated cirrhosis. These patients were evaluated at Cimeq's hospital. Forty-eight patients were studied. The variables studied were: progression of esophageal varices and spleen size, control of the etiology, phase of the disease and survival free of complications. Results: The control of the etiology was in 22 patients. Of the latter, 19 remained in the compensated phase. The average survival time free of major complications in patients with controlled etiology was 17 years. Conclusions: The progression of portal hypertension and the permanence of patients in the compensated phase of the disease were related to the control of the etiology. The highest complication-free survival was significantly greater in patients with control of the etiology of cirrhosis.

Key words: Liver cirrhosis. Portal hypertension. Control of the etiology.

\footnotetext{
Correspondencia:

*Zucel D. Cruz-Hernández

E-mail: zucelcruz83@gmail.com

Fecha de recepción: 27-05-2020

Fecha de aceptación: 03-09-2020

DOI: 10.24875/RHJM.20000065

Disponible en internet: 14-01-2021 Rev Hosp Jua Mex. 2020;87(4):181-187

www.revistahospitaljuarez.com 1405-9622/@ 2020 Sociedad Médico-Quirúrgica del Hospital Juárez de México, A.C. Publicado por Permanyer. Este es un artículo open access bajo la licencia CC BY-NC-ND (http://creativecommons.org/licenses/by-nc-nd/4.0/).
} 


\section{Introducción}

En la actualidad, uno de los principales problemas de salud en el mundo lo constituye la cirrosis hepática $(\mathrm{CH})^{1}$, consolidándose dentro de las 10 primeras causas de mortalidad general en países con ingreso medio bajo ${ }^{2}$. En Cuba se encuentra en la novena causa de muerte, según el anuario estadístico de salud ${ }^{3}$.

La historia natural de la $\mathrm{CH}$ se caracteriza por la progresión de la fase compensada a la descompensada, que ocurre entre los cinco a siete años, con el desarrollo de complicaciones secundarias a hipertensión portal (HTP) e insuficiencia hepática ${ }^{4-6}$.

El tratamiento antiviral con fármacos orales, desde finales de 2014, ha supuesto un gran avance en el campo de la hepatología. En los pacientes con $\mathrm{CH}$ por virus de la hepatitis $\mathrm{B}(\mathrm{VHB})$ y $\mathrm{C}(\mathrm{VHC})$ los tratamientos, además de inhibir la replicación viral, tienen un impacto evidente sobre la historia natural de la cirrosis tanto compensada como descompensada ${ }^{7-11}$.

Otra de las causas es la cirrosis por hepatopatías autoinmunes, donde el tratamiento inmunosupresor oportuno permite detener o retrasar la evolución de la enfermedad a estadios más avanzados ${ }^{12}$. Igualmente se ha observado en la cirrosis alcohólica, en donde el abandono del alcohol es la clave para el tratamiento ${ }^{13}$.

El tratamiento etiológico es vital, los pacientes de elección deben ser tratados de manera precoz, pues se ha demostrado que esto puede disminuir la progresión de la fibrosis ${ }^{14}$.

El objetivo del presente trabajo fue evaluar el impacto que tiene el control etiológico de la cirrosis en la evolución clínica de estos pacientes.

\section{Material y métodos}

Se realizó un estudio descriptivo, retrospectivo y longitudinal en pacientes con $\mathrm{CH}$ compensada, evaluados inicialmente por el Grupo de Hepatología del Hospital Cimeq, entre enero del 2016 y diciembre del 2018, donde de 287 pacientes cirróticos, 48 cumplieron con los criterios de inclusión.

\section{Criterios de inclusión}

Pacientes con $\mathrm{CH}$ confirmada por laparoscopia, biopsia hepática o ecografía abdominal, que se encontraban en fase compensada en la primera evaluación, con seguimiento evolutivo durante al menos dos años, con endoscopia digestiva superior y ecografía abdominal con Doppler esplenoportal al inicio y al final de la evaluación.

\section{Criterios de exclusión}

Pacientes con $\mathrm{CH}$ de etiología no precisada, mujeres embarazadas y pacientes que se negaron a participar en el estudio.

De los 48 enfermos que integraron el estudio, 30 $(62.5 \%)$ fueron del sexo femenino y $18(37.5 \%)$ del masculino. La media de seguimiento fue de 6 años, con una desviación estándar de 2.5 años.

Previo consentimiento informado, se recogieron los datos en correspondencia con los objetivos de la investigación, y con los elementos del interrogatorio se evaluó el desarrollo de complicaciones. Los pacientes fueron clasificados según los estadios de la $\mathrm{CH}$ del Consenso Baveno V, como se muestra en la tabla $1^{15}$. Además se identificaron los pacientes que se encontraban con control de la etiología de la cirrosis.

\section{Selección de variables}

\section{VARIABLES RELACIONADAS CON LAS MANIFESTACIONES de la Hipertensión Portal}

Se tomó en cuenta la progresión de várices esofágicas: se determinó su presencia o no según la endoscopia digestiva superior realizada y se estadificaron en ausentes, pequeñas o grandes; se describió como igual las que se mantuvieron en igual estadio, y como en desarrollo a los pacientes que anteriormente no tenían várices y las desarrollaron, así como las que progresaron de pequeñas a grandes.

Además, se analizó el tamaño del bazo y se consideró esplenomegalia cuando el diámetro del bazo medía más de 120 mm por ecografía abdominal. Para su evaluación se clasificaron en ausente y presente; se describió como igual a los pacientes que permanecieron sin esplenomegalia al final de la evaluación y como en desarrollo a los que iniciaron sin esplenomegalia y en la evaluación final estaba presente.

\section{Clasificación de los enfermos en CirRosis Hepática Compensada o Descompensada}

Según el consenso de Baveno $\mathrm{V}$, al inicio y al final de la evaluación. Descrita anteriormente. 
Tabla 1. Clasificación de la cirrosis hepática según las fases de la enfermedad, emitida en el consenso de Baveno V

\begin{tabular}{|l|c|l|l|}
\hline \multicolumn{3}{|c|}{ Clasificación de la cirrosis hepática según las fases de la enfermedad, emitida en el consenso de Baveno V } \\
\hline Fase & Estadios & Definición \\
\hline Compensada & 1 & Pacientes sin várices esofágicas y/o esofagogástricas sin ascitis u otra complicación \\
\hline Descompensada & 2 & Pacientes con várices esofágicas y/o esofagogástricas, sin sangrado, ascitis ni otra complicación \\
\hline & 4 & Pacientes con sangrado variceal, sin ascitis ni otra complicación \\
\hline & 5 & $\begin{array}{l}\text { Pacientes con ascitis y/o hepatocarcinoma, peritonitis bacteriana espontánea, íctero sin sangrado } \\
\text { variceal }\end{array}$ \\
\hline & $\begin{array}{l}\text { Pacientes con ascitis y sangrado por várices esofágicas, con o sin encefalopatía hepática, } \\
\text { hepatocarcinoma, peritonitis bacteriana espontánea o ictericia }\end{array}$ \\
\hline
\end{tabular}

Tabla 2. Progresión de las várices esofágicas en relación con el control de la etiología

\begin{tabular}{|c|c|c|c|c|c|c|c|}
\hline \multicolumn{8}{|c|}{ Progresión de las várices esofágicas en relación con el control de la etiología } \\
\hline & & \multicolumn{4}{|c|}{ Control de la etiología } & \multicolumn{2}{|c|}{ Total } \\
\hline & & \multicolumn{2}{|c|}{ No } & \multicolumn{2}{|c|}{ Sí } & \multirow[t]{2}{*}{ n } & \multirow[t]{2}{*}{ (\%) } \\
\hline & & n & (\%) & n & $(\%)$ & & \\
\hline \multirow[t]{2}{*}{ Progresión de las várices esofágicas } & Igual & 9 & 20.9 & 18 & 41.9 & 27 & 62.8 \\
\hline & Progresión & 13 & 30.2 & 3 & 6.9 & 16 & 37.2 \\
\hline Total & & 22 & 51.1 & 21 & 48.8 & 48 & $(100)$ \\
\hline
\end{tabular}

Relación entre la progresión o no de las várices con el control de la etiología $\mathrm{p}=0.02$.

5 pacientes fueron excluidos por recibir terapéutica endoscópica.

\section{Control de la etiología}

Según los siguientes criterios:

- Alcohólica: pacientes con más de seis meses de abstinencia de bebidas alcohólicas.

- VHB: en pacientes sin replicación viral (ADN VHB negativo).

- VHC: en pacientes sin replicación viral (ARN VHC negativo).

- Otras causas (hepatopatías autoinmunes, enfermedades colestásicas y enfermedad de Wilson): en pacientes con buena respuesta al tratamiento.

\section{SUPERVIVENCIA LIBRE DE COMPLICACIONES MAYORES}

Tiempo de supervivencia libre de complicaciones mayores en relación con el año del diagnóstico.

\section{Análisis estadístico}

Los datos se procesaron con el paquete estadístico SPSS versión 19.0 sobre Windows. Los resultados se compararon utilizando la prueba de McNemar. En el análisis entre las fases de la enfermedad, se utilizó el test de los signos y rangos de Wilcoxon. El análisis de supervivencia se realizó mediante las curvas de Kaplan-Meier, se tomaron los puntos de corte a los 5 y 10 años. Los resultados se exponen en tablas y gráficos.

\section{Resultados}

La tabla 2 muestra que de $22(51.1 \%)$ pacientes que no presentaban control de la etiología, $13(30.2 \%)$ desarrollaron las várices esofágicas y 9 (20.9\%) permanecieron en igual estadio, mientras que de 21 (48.8\%) pacientes con control de la etiología, solo en $3(6.9 \%)$ hubo desarrollo de las várices esofágicas $(p=0.002)$.

En la tabla 3 se observa que de $26(54.1 \%)$ pacientes sin control de la etiología, 16 (33.3\%) presentaron aumento de las dimensiones del bazo y solo 10 permanecieron igual. De los casos con la etiología 
Tabla 3. Progresión del bazo en relación con el control de la etiología

\begin{tabular}{|c|c|c|c|c|c|c|c|}
\hline & & \multicolumn{4}{|c|}{ Control de la etiología } & \multicolumn{2}{|c|}{ Total } \\
\hline & & \multicolumn{2}{|c|}{ No } & \multicolumn{2}{|c|}{ Sí } & \multirow[t]{2}{*}{ n } & \multirow[t]{2}{*}{$(\%)$} \\
\hline & & n & $(\%)$ & $\mathbf{n}$ & $(\%)$ & & \\
\hline \multirow[t]{2}{*}{ Progresión del bazo } & Igual & 10 & 20.8 & 18 & 37.5 & 28 & 62.8 \\
\hline & Progresión & 16 & 33.3 & 4 & 8.4 & 20 & 37.2 \\
\hline Total & & 26 & 54.1 & 22 & 45.9 & 48 & 100 \\
\hline
\end{tabular}

Relación entre la progresión o no de las várices con el control de la etiología p = 0.02 .

Tabla 4. Relación entre el control de la etiología y la evolución clínica de los pacientes al final del estudio

\begin{tabular}{|l|c|c|c|c|c|c|}
\multirow{2}{*}{$\begin{array}{l}\text { Fase de la } \\
\text { enfermedad }\end{array}$} & \multicolumn{3}{|c|}{ Control de la etiología } & \multicolumn{2}{c|}{ Total } \\
\cline { 2 - 6 } & \multicolumn{2}{|c|}{ No } & \multicolumn{2}{|c|}{ Sín } & \multirow{2}{*}{ n } & (\%) \\
\cline { 2 - 7 } & $\mathbf{n}$ & $(\%)$ & $\mathbf{n}$ & $(\%)$ & & \\
\hline Compensada & 11 & 42.3 & 19 & 86.3 & 30 & 58.3 \\
\hline Descompensada & 15 & 57.7 & 3 & 13.3 & 18 & $(37.5)$ \\
\hline Total & 26 & 54.1 & 22 & 45.9 & 48 & $(100)$ \\
\hline
\end{tabular}

Relación entre la progresión o no de las várices con el control de la etiología $\mathrm{p}=0.02$.

Tabla 5. Tiempo de supervivencia libre de complicaciones mayores en relación con el año del diagnóstico

\begin{tabular}{|l|c|}
\hline $\begin{array}{l}\text { Pacientes sin complicaciones } \\
\text { mayores (\%) }\end{array}$ & $\begin{array}{c}\text { Tiempo de supervivencia } \\
\text { libre de complicaciones }\end{array}$ \\
\hline 93.8 & 5 años \\
\hline 87.1 & 10 años \\
\hline
\end{tabular}

controlada, el mayor número de pacientes (18) se mantuvo igual y solo en 4 hubo aumento del tamaño del bazo $(p=0.02)$.

La tabla 4 muestra que en la evaluación final de 26 pacientes que no tenían control de la etiología, 15 (31.2\%) evolucionaron a la descompensación y 11 (22.9\%) se mantuvieron compensados. Mientras que de 22 pacientes (45.9\%) que presentaban control de la etiología de la enfermedad hepática, permanecieron 19 $(39.6 \%)$ en la fase compensada de la enfermedad y solo tres pacientes (6.3\%) evolucionaron a la descompensación. Se observó relación significativa $(p=0.002)$ entre el control de la etiología y la evolución de los pacientes.
Tabla 6. Tiempo de supervivencia libre de complicaciones mayores según el control de la etiología

\begin{tabular}{|l|c|c|}
\hline \multirow{2}{*}{$\begin{array}{l}\text { Control de la } \\
\text { etiología de la CH }\end{array}$} & \multicolumn{2}{|c|}{ Pacientes sin complicaciones mayores (\%) } \\
\cline { 2 - 3 } & $\mathbf{5}$ años & $\mathbf{1 0}$ años \\
\hline Controlada & 95.5 & 89.5 \\
\hline No controlada & 84.3 & 79.8 \\
\hline
\end{tabular}

Relación entre la progresión o no de las várices con el control de la etiología $\mathrm{p}<0.001$. $\mathrm{CH}$ : cirrosis hepática.

El tiempo promedio de supervivencia libre de complicaciones mayores fue de 13.5 años, con un intervalo de confianza (IC) de 11.4-15.6 años, como se muestra en la figura 1 y la tabla 5 . A los 5 y a los 10 años del diagnóstico de la cirrosis, el 93.8 y el $87.1 \%$ de los pacientes, respectivamente, no habían presentado complicaciones mayores.

En la figura 2 y la tabla 6 se observa que el tiempo promedio de supervivencia libre de complicaciones mayores de los pacientes con $\mathrm{CH}$ de etiología controlada fue de 17 años, con un IC de 14.9-19 años; y en los pacientes sin control de la etiología fue de 9 años, con un IC de 6.9-11.1 años. A los 5 y 10 años del diagnóstico de la $\mathrm{CH}$ en los pacientes con control de la etiología, la supervivencia libre de complicaciones mayores fue del 95.5 y el $89.5 \%$ respectivamente; sin embargo, en los pacientes con la etiología no controlada fue del $84.3 \%$ a los 5 años y del $79.8 \%$ a los 10 años.

\section{Discusión}

El procedimiento clave para el diagnóstico de la HTP es la determinación del gradiente de presión de la vena porta. Sin embargo, existen otros indicadores que orientan al diagnóstico y pronóstico de la 


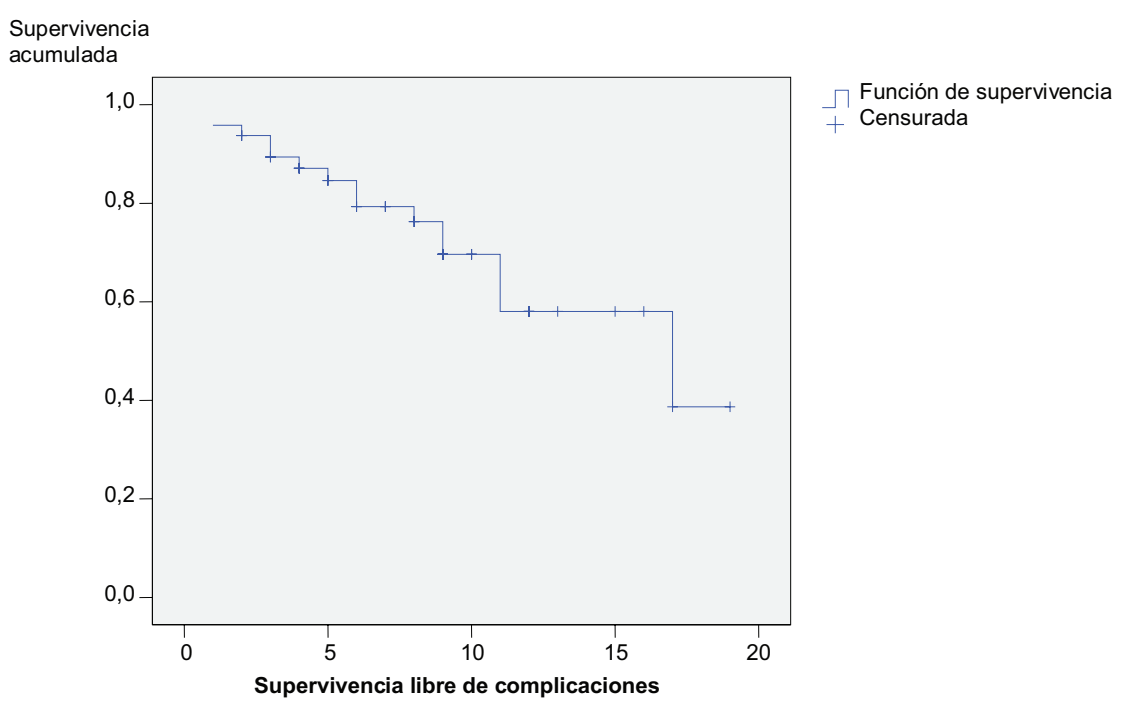

Figura 1. Tiempo de supervivencia libre de complicaciones mayores en relación con el año del diagnóstico.

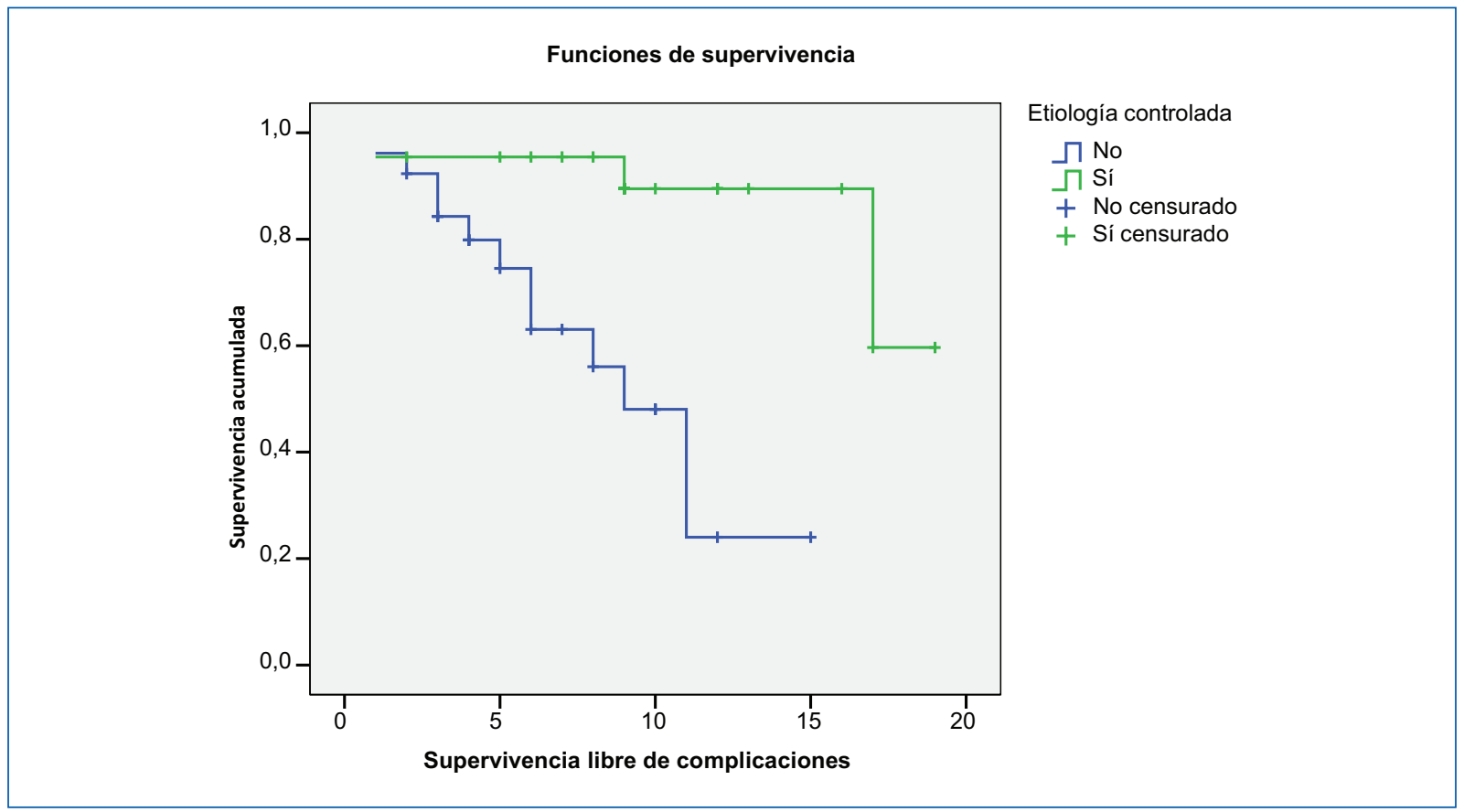

Figura 2. Tiempo de supervivencia libre de complicaciones mayores según el control de la etiología.

HTP, como algunas manifestaciones clínicas, ecográficas y endoscópicas. En la fase compensada con frecuencia se observa esplenomegalia y várices esofágicas y varios autores plantean el control de la etiología como factor pronóstico de su progresión ${ }^{16,17}$.
Los autores de esta investigación reportan la relación significativa entre el control de la etiología y la progresión de la HTP, teniendo en cuenta las várices esofágicas y la presencia de esplenomegalia.

Varios estudios reportan, en pacientes con $\mathrm{CH}$ por VHC tratados con respuesta viral sostenida (RVS), no 
solo la no progresión de la HTP, sino la reducción de esta $^{7,18,19}$.

De estos resultados se desprende que la $\mathrm{CH}$ es un proceso reversible si se elimina el factor que la causa, aunque esto no sucede en todos los pacientes, bien sea porque se requiere de más tiempo o porque en algunos la hepatopatía se encuentra en estadios muy avanzados?

El tratamiento con antivirales en los pacientes con cirrosis por VHB y VHC ha revolucionado la evolución de esta enfermedad, pues la posibilidad de regresión de la fibrosis en estadios iniciales y recuperación de la función hepática es mayor ${ }^{20}$.

En un estudio realizado en 1,323 pacientes con $\mathrm{CH}$ por VHC se evidencia diferencia altamente significativa en la evolución a la fase descompensada de la enfermedad entre los pacientes con RVS y los que no ${ }^{21}$; similares resultados obtuvieron Fattovich, et al. ${ }^{22}$ y el presente estudio.

En una revisión de 92 estudios realizada por D’Amico, et al., el tratamiento etiológico se asoció a una reducción significativa del riesgo de descompensación ${ }^{14}$. Similares resultados han sido reportados por otros autores ${ }^{23}$.

Está bien establecido que en las hepatopatías autoinmunes el tratamiento inmunosupresor oportuno permite detener o retrasar la evolución de la enfermedad a estadios más avanzados, principalmente en la hepatitis autoinmune. En la serie publicada por Rodríguez, et al. ${ }^{12}$ en 24 pacientes evaluados, 14 (58\%) pacientes con $\mathrm{CH}$ compensada de etiología autoinmune y respuesta al tratamiento no tuvieron progresión de la enfermedad.

En la presente investigación, el tiempo promedio de supervivencia libre de grandes complicaciones a partir del diagnóstico de la enfermedad fue de 13.5 años; otros autores reportan menos tiempo, pero no se analizó la supervivencia con relación a la fecha del diagnóstico, sino a la fecha de la primera evaluación ${ }^{22}$.

Varios estudios recogen una supervivencia libre de complicaciones significativamente mayor en pacientes cirróticos con control de la etiología ${ }^{21-23}$, similar a lo hallado en el presente estudio.

Estos resultados muestran la influencia determinante del control de la etiología en la evolución de los pacientes.

\section{Conclusiones}

El control de la etiología en la $\mathrm{CH}$ disminuye la progresión de la HTP y aumenta sustancialmente la permanencia de los pacientes en la fase compensada de la enfermedad. La supervivencia libre de complicaciones mayores fue significativamente mayor en los pacientes con etiología controlada.

\section{Financiamiento}

Los autores no recibieron patrocinio para llevar a cabo este artículo.

\section{Conflicto de intereses}

Los autores declaran no tener conflicto de intereses alguno.

\section{Responsabilidades éticas}

Protección de personas y animales. Los autores declaran que para esta investigación no se han realizado experimentos en seres humanos ni en animales.

Confidencialidad de los datos. Los autores declaran que han seguido los protocolos de su centro de trabajo sobre la publicación de datos de pacientes.

Derecho a la privacidad y consentimiento informado. Los autores han obtenido el consentimiento informado de los pacientes y/o sujetos referidos en el artículo. Este documento obra en poder del autor de correspondencia.

\section{Bibliografía}

1. Zubieta-Rodríguez R, Gómez-Correa J, Rodríguez-Amaya R, Ariza-Mejía K, Toloza-Cuta NA. Mortalidad hospitalaria en pacientes cirróticos en un hospital de tercer nivel. Rev Gastroenterol Mex. 2017;82(3):203-9.

2. Las 10 causas principales de defunción en el mundo [Internet]. Ginebra: Organización Mundial de la Salud; mayo del 2018. Disponible en: http:// www.who.int/es/news-room/fact-sheets/detail/the-top-10-causes-of-death

3. Estadísticas de Salud en Cuba. Anuario Estadístico de Salud 2017 [Base de datos en Internet]. Cuba: Biblioteca virtual en salud. Disponible en: http://files.sld.cu/dne/files/2018/04/Anuario Electrónico-Espa\%C3\%B10l-2017-ed-2018.pdf

4. Bernal W, Jalan R, Quaglia A, Simpson K, Wendon J, Burroughs A. Acute-on-chronic liver failure. Lancet. 2015;386:1576-87.

5. Tapper EB, Parikh ND. Mortality due to cirrosis and liver cancer in the United States, 1999-2016: observational study. BMJ. 2018;362:k2817.

6. Bernardi M, Caraceni P. Novel perspectives in the management of decompensated cirrhosis. Nat Rev Gastroenterol Hepatol. 2018;15:753-64.

7. García-Pagán JC, Sabela L, Alvarado-Tapias E, Mariño Z, Londoño MC, Llop $E$, et al. El tratamiento antiviral para la hepatitis $C$ reduce la hipertensión portal en pacientes con cirrosis. Gastroenterol. 2017;153(5):1273-83.

8. Salmon D, Mondelli MU, Maticic M, Arends JE. The benefits of hepatitis C virus cure: every rose has thorns. J Hepatol. 2018;25:320-8.

9. Hsu YC, Ho HJ, Huang YT, Wang HH, Wu MS, Lin JT, et al. Association between antiviral treatment and extrahepatic outcomes in patients with hepatitis C virus infection. Gut. 2015;64:495-503.

10. Terrault NA, Lok ASF, McMahon BJ, Chang KM, Hwang JP, Jonas MM, et al. Update on prevention, diagnosis, and treatment of chronic hepatitis B: AASLD 2018 hepatitis B guidance. Hepatology. 2018;67:1560-99.

11. Asociación Europea para el Estudio del Hígado. EASL 2017. Guías de práctica clínica sobre el manejo del virus de la hepatitis B. J Hepatol. 2017:67:370-98

12. Rodríguez Rodríguez $\mathrm{H}$, Samada Suárez M, Hernández Perera JC, Pérez González T, Cruz Hernández ZD, Chao González L. Evolución de pacientes con hepatitis autoinmune después de tratamiento. Invest Medicoquir. 2016;8(1):4-12.

13. Wang $X$, Wu $B$. Critical issues in the diagnosis and treatment of liver cirrhosis. Gastroenterol Rep (Oxf). 2019;7(4):227-30. 
14. D'Amico G, Perricone G. Prediction of descompensation in patients with compensated cirrhosis: Does Etiology Matter? Curr Hepatology Rep. 2019;18:144-56.

15. De Franchis R. Revising consensus in portal hypertension: Report of the Baveno $\mathrm{V}$ consensus workshop on methodology of diagnosis and therapy in portal hypertension. J Hepatol. 2010;53(4):762-8.

16. Anthony PP, Ishak KG, Nayak NC, Poulsen HE, Scheuer PJ, Sobin LH The morphology of cirrhosis. Recommendations on definition, nomenclature, and classification by a working group sponsored by the World Health Organization. J Clin Pathol. 1978;31(5):395-414.

17. Sarrazin C, Berg T, Ross RS, Schirmacher P, Wedemeyer H. Prophylaxis, diagnosis and therapy of hepatitis $\mathrm{C}$ virus (HCV) infection: the German guidelines on the management of HCV infection. Z Gastroenterol. 2010;48:289-351.

18. Mauro E, Crespo G, Montironi C, Londoño MC, Hernández-Gea V, Ruiz $\mathrm{P}$, et al. Mediciones de presión portal y rigidez hepática en la predicción de la regresión de la fibrosis después de una respuesta virológica sostenida en la hepatitis C recurrente. Hepatol. 2017;67(5):1683
19. Mandorfer M, Kozbial K, Schwabl P, Freissmuth C, Schwarzer R Stern $R$, et al. Sustained virologic response to interferon-free therapies ameliorates HCV-induced portal hypertension. J Hepatol. 2016;65(4): 692-99.

20. Mutimer DJ, Lok A. Management of HBV- and HCV-induced end stage liver disease. Gut. 2012;61(1):59-67.

21. Nahon $P$, Valerie $M$, Bourcier $V$. Eradication of hepatitis $C$ virus infection in patients with cirrhosis reduces risk of liver and non-liver complications. Gastroenterol. 2017;152(1):142-56.

22. Fattovich G, Pantalena M, Zagni I, Realdi G, Solko W, Christensen E, and the European Concerted Action on Viral Hepatitis(EUROHEP). Effect of hepatitis $B$ and $C$ virus infections on the natural history of compensated cirrhosis: A cohort study of 297 patients Am J Gastroenterol. 2002;97(11):2886-95.

23. Hallager S, Laudelund S, Christensen PB, Kjaer M, Thorup Roege B, Elmergaard Gronbaek K, et al. Liver-related morbidity and mortality in patients with chronic hepatitis $C$ and cirrhosis with and without sustained virologic response. Clin Epidemiol. 2017;9:501-16. 